Article

\title{
A Note on Superspirals of Confluent Type
}

\author{
Jun-ichi Inoguchi ${ }^{1, *,+} \mathbb{\infty}$, Rushan Ziatdinov ${ }^{2,+} \mathbb{C}$ and Kenjiro T. Miura ${ }^{3,+}+\mathbb{C}$ \\ 1 Institute of Mathematics, University of Tsukuba, Tsukuba 305-8571, Japan \\ 2 Department of Industrial Engineering, Keimyung University, Daegu 704-701, Korea; ziatdinov@kmu.ac.kr or \\ ziatdinov.rushan@gmail.com \\ 3 Department of Mechanical Engineering, Shizuoka University, Hamamatsu 432-8561, Japan; \\ miura.kenjiro@shizuoka.ac.jp \\ * Correspondence: inoguchi@math.tsukuba.ac.jp \\ + These authors contributed equally to this work.
}

Received: 13 April 2020; Accepted: 6 May 2020; Published: 11 May 2020

check for updates

\begin{abstract}
Superspirals include a very broad family of monotonic curvature curves, whose radius of curvature is defined by a completely monotonic Gauss hypergeometric function. They are generalizations of log-aesthetic curves, and other curves whose radius of curvature is a particular case of a completely monotonic Gauss hypergeometric function. In this work, we study superspirals of confluent type via similarity geometry. Through a detailed investigation of the similarity curvatures of superspirals of confluent type, we find a new class of planar curves with monotone curvature in terms of Tricomi confluent hypergeometric function. Moreover, the proposed ideas will be our guide to expanding superspirals.
\end{abstract}

Keywords: superspiral; hypergeometric function; log-aesthetic curve; similarity geometry; aesthetic shape modeling; CAD

\section{Introduction}

Log-aesthetic curves (LAC) were proposed a decade ago to meet the requirements of industrial design to produce visually pleasing shapes. Followed by the discovery of superspirals, curves with monotonic curvature are now considered as an excellent tool for generating highly-smooth shapes which are useful in computer aided design and styling. Such non-polynomial curves are determined in terms of complex special functions and can be precisely computed in modern computer algebra systems and programming languages.

LAC form a one-parameter family of curves parametrized by the slope [1,2]. The LAC of slope 1 is the logarithmic spiral. As a generalization of logarithmic spiral, the second named author introduced a new class of fair curves-the superspiral of confluent type [3]. The superspiral of confluent type is a planar curve whose radius of curvature is a Kummer's hypergeometric function of confluent type (see (6)). The family of superspirals of confluent type is fairly large and includes logarithmic spirals as a particular example. However it is unclear how to describe the distribution of superspirals of confluent type in the whole family of those. In particular, the location of the logarithmic spiral in the whole family is not clear. To overcome these difficulties, we use a new framework "similarity geometry" for the study of aesthetic curves developed in our previous works $[4,5]$. The usage of the log-aesthetic curve for practical design is still limited and we should extend its formula to obtain various curves to solve practical design problems, such as $G^{n}$ Hermite interpolation, deformation and smoothing; data-point fitting; and blending plural curves [5]. We should explore other types of curves in a systematic way via similarity geometry.

In this paper we give an explicit Formula (13) of similarity curvature for superspirals of confluent type. The formula (Equation(13)) clarifies the precise location of logarithmic spiral in the whole family 
of superspirals of confluent type. The present study implies that planar curves whose radii of curvature are Tricomi's hypergeometric function of confluent type are new candidates of fair curves in industrial shape design. We close this paper by exhibiting some pictures of those curves.

\section{Preliminaries}

Let us consider a Riccati differential equation [6]:

$$
\frac{d z}{d x}=p(x)+2 q(x) z+r(x) z^{2}
$$

where $q(x) \neq 0$ and $r(x) \neq 0$. Assume that we know a particular solution $u(x)$ to Equation (1). After a substitution

$$
z=u+\frac{1}{y}
$$

Equation (1) turns into

$$
\frac{d y}{d x}+2 y(q(x)+r(x) u(x))=-r(x) .
$$

Hence by solving linear ordinary differential equation (ODE) (2), we obtain a general solution in the form of

$$
y(x)=\left(\int r(x) \exp \left[2 \int f(x) d x\right] d x+C\right) \times \exp \left[-2 \int f(x) d x\right],
$$

where $f(x)=q(x)+r(x) u(x)$ and $C$ is an integral constant.

The Kummer confluent hypergeometric function [7] is defined by

$$
{ }_{1} F_{1}(a, c ; x)=\sum_{n=0}^{\infty} \frac{(a)_{n}}{(c)_{n} n !} x^{n},
$$

where $(a)_{n}=a(a+1) \cdots(a+n-1)$ for $n>0$. For avoiding the case of $(c)_{n}=0$, we assume that $c \notin \mathbb{Z}_{\leq 0}$. For the case of $a=c$, Equation (4) becomes

$$
{ }_{1} F_{1}(a, a ; x)=\sum_{n=0}^{\infty} \frac{(a)_{n}}{(a)_{n} n !} x^{n}=\sum_{n=0}^{\infty} \frac{1}{n !} x^{n}=e^{x} .
$$

The confluent hypergeometric function ${ }_{1} F_{1}(a, c ; x)$ can be also denoted as $M(a, c, x)$.

Definition 1. The Tricomi confluent hypergeometric function $U(a, c, x)[7]$ is defined by

$$
U(a, c, x)=\frac{\Gamma(1-c)}{\Gamma(a+1-c)}{ }_{1} F_{1}(a, c, x)+\frac{\Gamma(c-1)}{\Gamma(a)} x^{1-c}{ }_{1} F_{1}(a+1-c, 2-c, x),
$$

where $\Gamma(x)$ is Gamma function [7].

The functions $M(a, c, x)$ and $U(a, c, x)$ are the fundamental solutions of Kummer's confluent hypergeometric differential equation [8]:

When $a=c \notin \mathbb{Z}_{\leq 0}$, we get

$$
U(a, a, x)=\Gamma(1-a) e^{x}+\Gamma(a-1) x^{1-a}{ }_{1} F_{1}(1,2-a, x) .
$$

Definition 2. The Whittaker M-function and W-function [8] are defined by

$$
\begin{aligned}
& M(\mu, v, z)=e^{-z / 2} z^{v+\frac{1}{2}}{ }_{1} F_{1}\left(v-\mu+\frac{1}{2}, 2 v+1 ; z\right), \\
& W(\mu, v, z)=e^{-z / 2} z^{v+\frac{1}{2}} U\left(v-\mu+\frac{1}{2}, 2 v+1 ; z\right) .
\end{aligned}
$$


It can be seen that $M(\mu, v, z)$ and $W(\mu, v, z)$ are sometimes denoted as $M_{v, \mu}(z)$ and $W_{v, \mu}(z)$ in various textbooks; e.g., [9] (pp. 339-351).

The $M$-function and $W$-function are the fundamental solutions to the Whittaker differential equation:

$$
\frac{d^{2} y}{d z^{2}}+\left(-\frac{1}{4}+\frac{\mu}{z}+\frac{\frac{1}{4}-v^{2}}{z^{2}}\right) y=0
$$

which is a modified form of the confluent hypergeometric equation. Thus the general solution to the Whittaker differential equation is expressed as

$$
y=a_{1} M(\mu, v, z)+a_{2} W(\mu, v, z)
$$

if $2 \mu \notin \mathbb{Z}$ (see [10]).

Suppose that $\mathbf{p}(\theta)$ is a superspiral [3] of confluent type with radius of curvature

$$
\rho(\theta)={ }_{1} F_{1}(a, c ; \lambda \theta) .
$$

Then $\rho$ satisfies the hypergeometric differential equation of confluent type:

$$
\theta \frac{d^{2} \rho}{d \theta^{2}}=a \lambda \rho-(c-\lambda \theta) \frac{d \rho}{d \theta}
$$

As we have mentioned in the preceding section, the general solution of this ODE includes two parameters (integral constants). Equation (5) implies that the general solution of (7) is given by

$$
\rho(a, c, \alpha, \beta, \lambda, \theta)=\alpha_{1} F_{1}(a, c, \lambda \theta)+\beta(\lambda \theta)^{1-c}{ }_{1} F_{1}(a+1-c, 2-c, \lambda \theta), \alpha, \beta \in \mathbb{R} .
$$

Here we recall the notion of similarity curvature from $[4,5]$.

Definition 3. The similarity curvature $S(\theta)$ of a planar curve $\mathbf{p}(\theta)$ parametrized by tangent angle $\theta$ is

$$
S(\theta)=-\frac{1}{\rho(\theta)} \frac{d \rho}{d \theta}(\theta) .
$$

Note that planar curves are determined by the similarity curvature uniquely up to similarity transformations. The similarity curvature $S(\theta)$ of a superspiral $\mathbf{p}(\theta)$ of confluent type satisfies the following Riccati equation:

$$
\frac{d S}{d \theta}=S^{2}-\frac{a \lambda}{\theta}-\frac{c-\lambda \theta}{\theta} S .
$$

It should be remarked that the above Riccati equation is of first order; thereforem its general solution contains one parameter (integral constant).

The general solution to the Riccati Equation (Equation (9)) is

$$
S(a, c, \alpha, \beta, \theta)=-\frac{1}{\rho(a, c, \alpha, \beta, \theta)} \frac{d}{d \theta} \rho(a, c, \alpha, \beta, \theta) .
$$

One can see that the similarity curvature $S$ is expressed by the Kummer confluent hypergeometric function and Tricomi confluent hypergeometric function (see [5] (p. 257)).

Hereafter we investigate planar curves determined by the similarity curvature (10). We know that ${ }_{1} F_{1}(a, c, \lambda \theta)$ and $(\lambda \theta)^{1-c}{ }_{1} F_{1}(a+1-c, 2-c, \lambda \theta)$ are fundamental solutions to (7). The former solution is nothing but the radius of curvature of the superspiral of confluent type (6). In other words, The similarity curvature of a superspiral of confluent type (6) is $S(a, c, 1,0, \theta)$.

The curvature monotonicity is the fundamental property for planar curves to be aesthetic or fair [11]. Here we examine two particular cases, $\alpha=0$ and $\beta=0$. 
1. The case wherein $\beta=0$.

The general solution (8) turns into $\rho(a, c, \alpha, \beta, \lambda, \theta)=\alpha_{1} F_{1}(a, c, \lambda \theta)$, which is a completely monotonic function for $c>a>0$ for $\lambda \theta \in(0,-\infty)$ [12]. Therefore we can consider this class of curves as fair curves.

2. The case in which $\alpha=0$.

Let us take in the account Lemma 1 (e.g., [13]) below to find the monotonicity conditions for this case. The monotonicity conditions for ${ }_{1} F_{1}(a-c+1,2-c, \lambda \theta)$ are $a<1, c<a+1$ for $\lambda \theta \in(0,-\infty)$ (Theorem 5 in [12]). Now we apply Lemma 1 to $f(\theta)=(\lambda \theta)^{1-c}$ with $\alpha=1-c \geq 1$ and $g(\theta)={ }_{1} F_{1}(a-c+1,2-c, \lambda \theta)$ with $\beta=1$; then $\rho(a, c, 0, \beta, \lambda, \theta)$ is monotonic if $1-c+1 \leq 1$; i.e., $c=1$. Therefore, the sufficient monotonicity condition for this case is $c=1$ and $0<a<1$ for $\lambda \theta \in(0,-\infty)$.

Lemma 1 ([13]). Let $f(x)$ and $g(x)$ be operator monotone functions. Then the following functions are also operator monotonous:

(i) $f(x)^{\alpha} g(x)^{\beta}$ for $\alpha, \beta \geq 0$ such that $\alpha+\beta \leq 1$;

(ii) $f\left(x^{-1}\right)^{-1}$.

Additionally, we are interested in the case $a=c$ for which we have

$$
\rho(\theta)=\exp (\lambda \theta)
$$

More precisely, the radius of curvature function is defined as $\rho(a, a, 1,0, \theta)$. The planar curve $\mathbf{p}(\theta)$ with the radius of curvature $\rho(a, a, 1,0, \theta)$ is a log-aesthetic curve of slope 1 with similarity curvature $S=-\lambda$; i.e., it is a logarithmic spiral.

Under the choice $a=c$, the Ricatti equation (9) becomes

$$
\frac{d S}{d \theta}=S^{2}-\frac{a \lambda}{\theta}-\frac{a-\lambda \theta}{\theta} S .
$$

One can check that $S=-\lambda$ (i.e., a log-aesthetic curve) is a particular solution to (11). The general solution is represented as

$$
S(a, a, \alpha, \beta, \theta)=-\frac{1}{\rho(a, a, \alpha, \beta, \theta)} \frac{d}{d \theta} \rho(a, a, \alpha, \beta, \theta),
$$

where

$$
\rho(a, a, \alpha, \beta, \theta)=\alpha e^{\lambda \theta}+\beta(\lambda \theta)^{1-a}{ }_{1} F_{1}(1,2-a, \lambda \theta)
$$

is a general solution to the $\operatorname{ODE}(7)$ with $a=c$. Let us look for the general solution to (11). Suppose that

$$
S=-\lambda+\frac{1}{y}
$$

Then $y$ satisfies

$$
y^{\prime}-\frac{a+\lambda \theta}{\theta} y=-1
$$

First, we consider a homogeneous ODE

$$
y^{\prime}-\frac{a+\lambda \theta}{\theta} y=0
$$

associated to (12). The general solution to this homogeneous ODE is given by 


$$
y=A e^{\lambda \theta} \theta^{a},
$$

where $A$ is a constant. We use the Lagrange's method of variation of constants [14] to solve this ODE.

Assuming that

$$
y=A(\theta) e^{\lambda \theta} \theta^{a}
$$

is a solution to (12), and then substituting $y=A(\theta) e^{\lambda \theta} \theta^{a}$ into the linear ordinary differential equation (ODE) (12), we get the 1st order ODE:

$$
A^{\prime}(\theta) e^{\lambda \theta} \theta^{a}=-1
$$

The general solution of this ODE is given by Whittaker $M$-function as

$$
A(\theta)=-\frac{1}{\lambda(1-a)} \theta^{-a}(\lambda \theta)^{a / 2} e^{-\lambda \theta / 2} M(-a / 2,-a / 2+1 / 2, \lambda)+C,
$$

where $C$ is an integral constant.

Hence the solution $y=A(\theta) e^{\lambda \theta} \theta^{a}$ to (12) contains two parameters $a$ and (integral constant) $C$.

Finally, the general solution $S$ to (11) is given by

$$
S(\theta ; a, C)=-\lambda\left(1+\frac{1-a}{(\lambda \theta)^{a / 2} e^{\lambda \theta / 2} \mathrm{M}(-a / 2,-a / 2+1 / 2, \lambda)+C \theta^{a} e^{\lambda \theta}}\right) .
$$

After defining $a=1$ in the general solution, we get the log-aesthetic curve with $S=-\lambda$. Equation (13) shows the location of logarithmic spiral in the family of superspirals of confluent type.

\section{Conclusions}

The log-aesthetic curve of slope 1 is characterized as a superspiral of confluent type determined by $\rho(1,1,1,0, \theta)=\exp (\lambda \theta)$ and it has the similarity curvature $S=-\lambda$. The family of superspirals of confluent type determined by $\rho(a, a, \alpha, \beta, \theta)$ contains log-aesthetic curve of slope 1 . It is strictly larger than the set of log-aesthetic curves of slope 1 . The superspirals of confluent type with $\rho(a, a, \alpha, \beta, \theta)$ satisfies the differential equation

$$
\frac{d S}{d \theta}=S^{2}-\frac{a \lambda}{\theta}-\frac{a-\lambda \theta}{\theta} S,
$$

whose general solution of the above equation is denoted by $S(a, a, \alpha, \beta, \theta)$. The log-aesthetic curve of slope 1 is characterized as $S(1,1, \alpha, \beta, \theta)=-\lambda$.

The solutions of (9) define a large class of planar curves, even if $a=c$. Kummer's equation (7) has 2-dimensional solution space which is spanned by ${ }_{1} F_{1}(a, c ; \lambda \theta)$ and $(\lambda \theta)^{1-c}{ }_{1} F_{1}(a-c+1,2-c ; \lambda \theta)$. The solution ${ }_{1} F_{1}(a, c ; x)$ defines the superspiral of confluent type. On the other hand, the solution $(\lambda \theta)^{1-c}{ }_{1} F_{1}(a-c+1,2-c ; \lambda \theta)$ induces another family of planar curves.

It would be interesting to investigate planar curve determined by $\rho(\theta)=(\lambda \theta)^{1-c}{ }_{1} F_{1}(a-c+1,2-$ $c ; \lambda \theta)$ or $\rho(\theta)=U(a, c, \lambda \theta)$.

We exhibit some pictures of planar curves with radius of curvature $\rho(\theta)=U(a, c, \lambda \theta)$. See Figures 1-7.

In our future works on monotone curvature curves, we plan to apply generalized hypergeometric function, $p F_{q}\left(a_{1}, \ldots, a_{p} ; b_{1}, \ldots, b_{q} ; z\right)$, the Meijer $G$-function which includes most of the known special functions as particular cases, and the Fox $H$-function, which is a generalization of the Meijer $G$-function. Moreover, Lemma 1 will allow us to expand the family of superspirals and add more degrees of freedom to curves. 


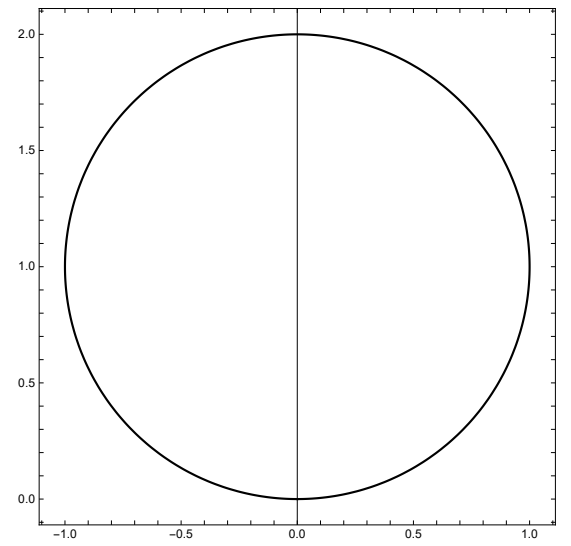

(a)

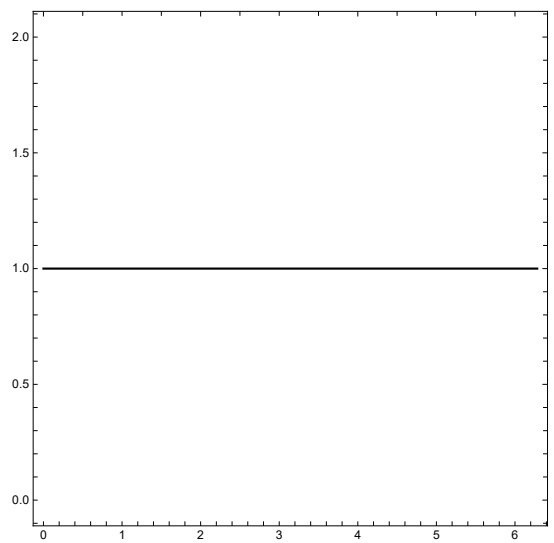

(b)

Figure 1. (a) Curve with $\rho(\theta)=U(0,1, \theta), 0 \leq \theta \leq 2 \pi$, (b) curvature.

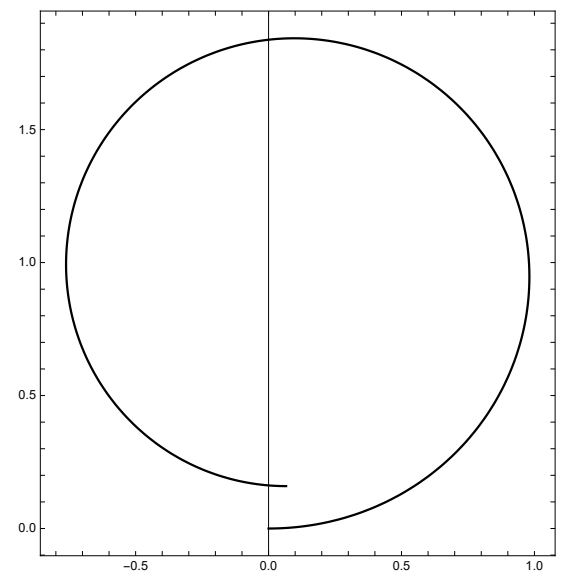

(a)

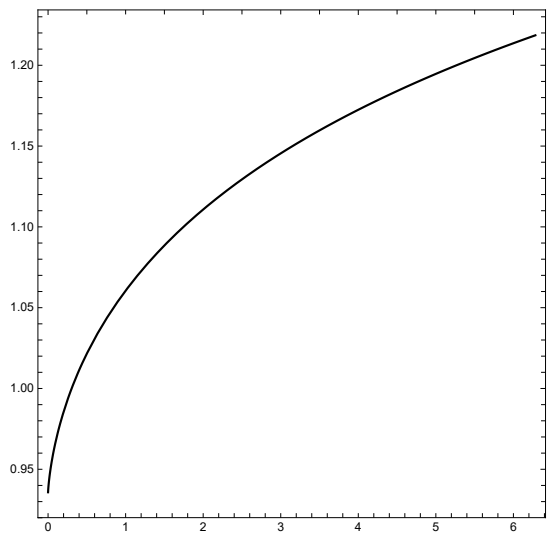

(b)

Figure 2. (a) Curve with $\rho(\theta)=U(0.1,0.1, \theta), 0 \leq \theta \leq 2 \pi$, (b) curvature.

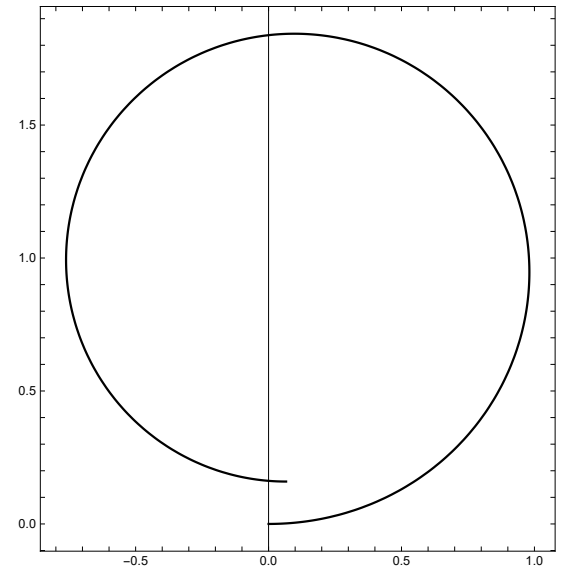

(a)

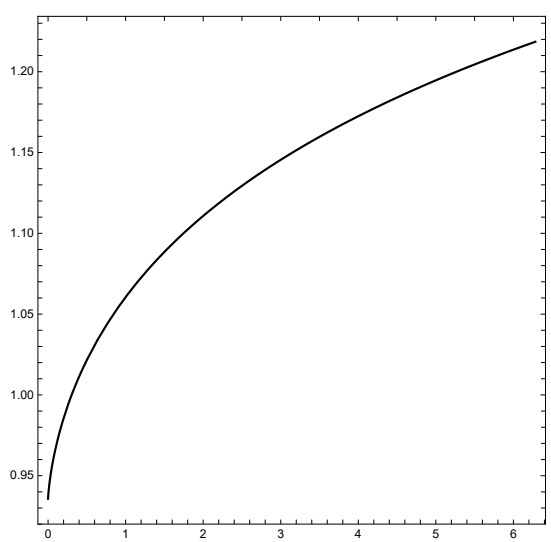

(b)

Figure 3. (a) Curve with $\rho(\theta)=U(0.1,0.5, \theta), 0 \leq \theta \leq 2 \pi$, (b) curvature. 


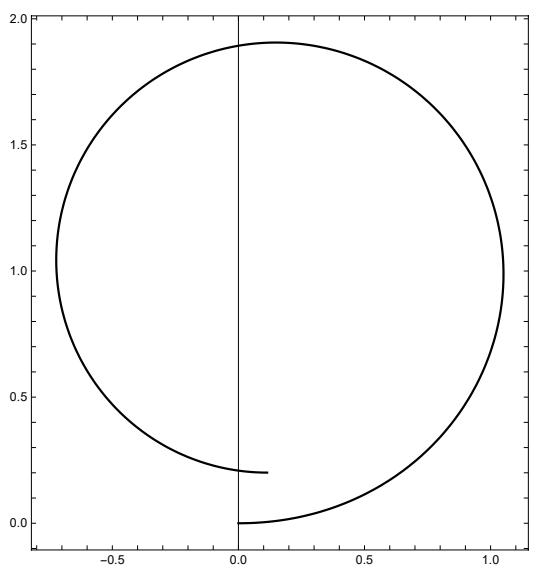

(a)

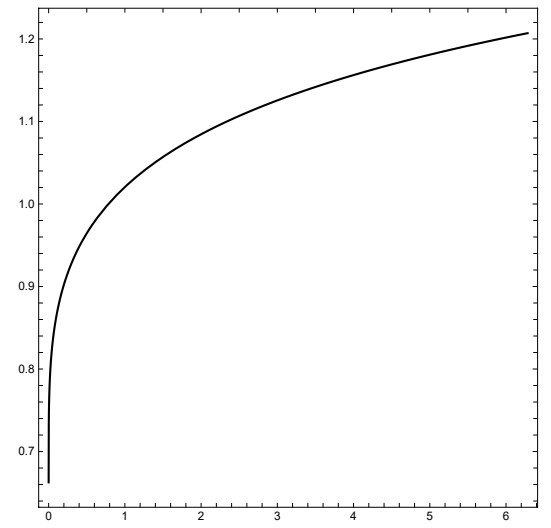

(b)

Figure 4. (a) Curv with $\rho(\theta)=U(0.1,0.8, \theta), 0 \leq \theta \leq 2 \pi$, (b) curvature.

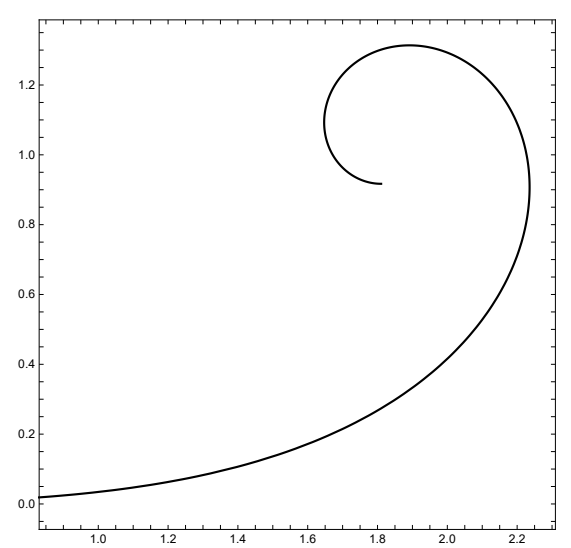

(a)

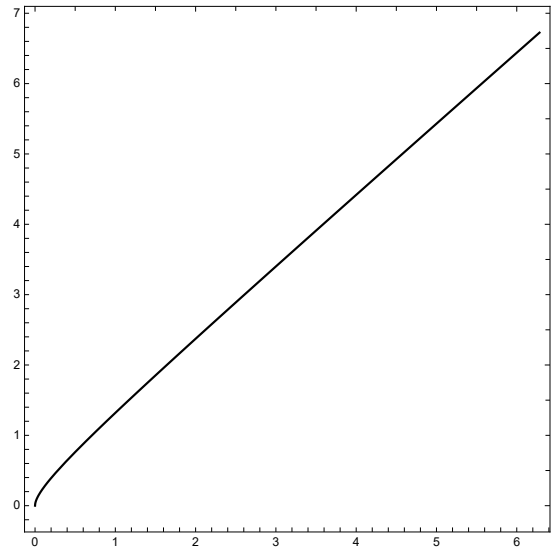

(b)

Figure 5. (a) Curve with $\rho(\theta)=U(1,1.5, \theta), 0 \leq \theta \leq 2 \pi$, (b) curvature.

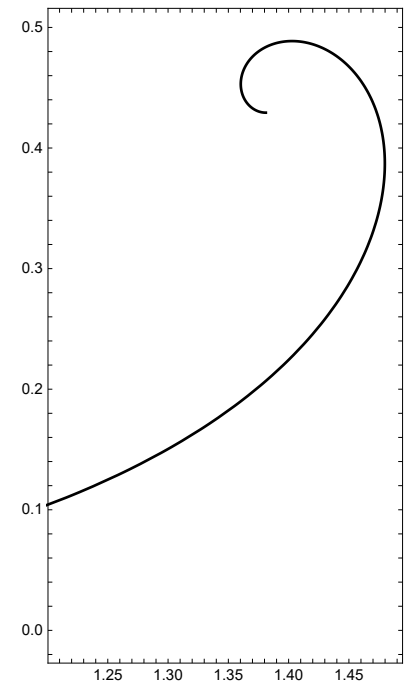

(a)

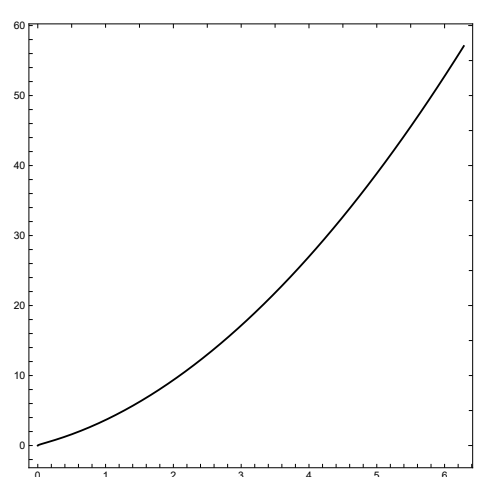

(b)

Figure 6. (a) Curve with $\rho(\theta)=U(2,1.5, \theta), 0 \leq \theta \leq 2 \pi$, (b) curvature. 


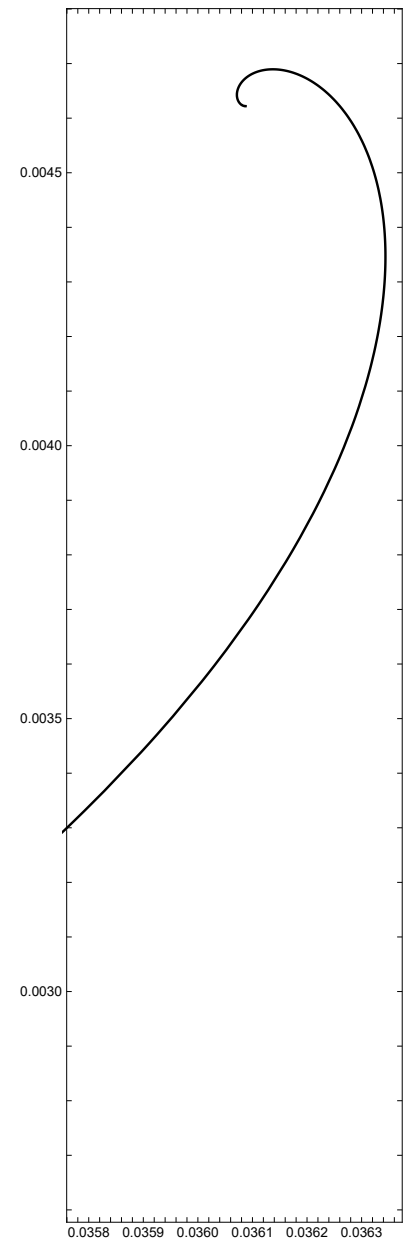

(a)

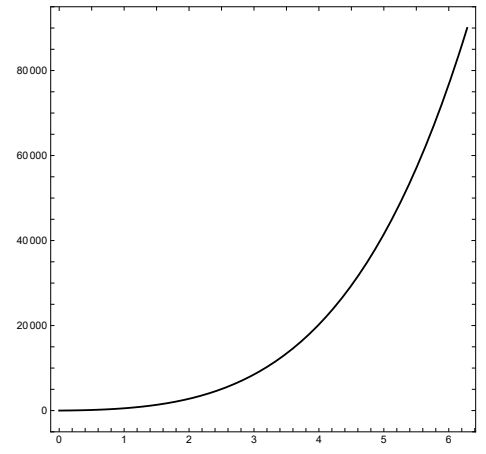

(b)

Figure 7. (a) Curve with $\rho(\theta)=U(5,1.5, \theta), 0 \leq \theta \leq 2 \pi$, (b) curvature.

Author Contributions: All authors have contributed equally to the study and preparation of the article. Conceptualization, J.-i.I., R.Z. and K.T.M.; Methodology, J.-i.I., R.Z. and K.T.M.; Validation, J.-i.I., R.Z. and K.T.M.; Investigation, J.-i.I., R.Z. and K.T.M.; Writing—original draft preparation, J.-i.I., R.Z. and K.T.M.; Writing-review and editing, J.-i.I., R.Z. and K.T.M. All authors have read and agreed to the published version of the manuscript.

Funding: This work is partly supported by JSPS KAKENHI grant numbers JP16H03941, JP19H02048, JP19K03461; and JST CREST grant number JPMJCR1911, 2019 IMI Joint Use Research Program Short-term Joint Research "Applications of discrete differential geometry to design: from theory to practice".

Conflicts of Interest: The authors declare no conflict of interest.

\section{References}

1. Miura, K.T. A general equation of aesthetic curves and its self-affinity. Comput. Aided Des. Appl. 2006, 3, 457-464. [CrossRef]

2. Ziatdinov, R.; Yoshida, N.; Kim, T. Analytic parametric equations of log-aesthetic curves in terms of incomplete gamma functions. Comput. Aided Geom. Des. 2012, 29, 129-140. [CrossRef]

3. Ziatdinov, R. Family of superspirals with completely monotonic curvature given in terms of Gauss hypergeometric function. Comput. Aided Geom. Des. 2012, 29, 510-518. [CrossRef]

4. Inoguchi, J. Attractive plane curves in differential geometry. In Mathematical Progress in Expressive Image Synthesis, 3rd ed.; Mathematics for Industry Vol 24; Springer: Singapore, 2016; pp. 121-135.

5. Inoguchi, J.; Ziatdinov, R.; Miura, K.T. Generalization of log-aesthetic curves via similarity geometry. Jpn. J. Ind. Appl. Math. 2019, 36, 239-259. [CrossRef]

6. Egorov, A.I. Riccati Equations; Pensoft Publishers: Sofia-Moscow, Bulgaria, 2007. 
7. Lebedev, N.N. Special Functions and their Applications; (translated by Silverman, R. A.); Dover Publications: Mineola, NY, USA, 1972.

8. Slater, L.J. Confluent Hypergeometric Functions; Cambridge University Press: Cambridge, UK, 1960.

9. Whittaker, E.T.; Watson, G.N. A Course in Modern Analysis, 4th ed.; Cambridge University Press: Cambridge, UK, 1990.

10. Zaitsev, V.F.; Polyanin, A.D. Handbook of Exact Solutions for Ordinary Differential Equations; CRC Press: Boca Raton, FL, USA, 2002.

11. Farin, G.; Sapidis, N. Curvature and the fairness of curves and surfaces. IEEE Comput. Graph. Appl. 1989, 9, 52-57. [CrossRef]

12. Miller, K.S.; Samko, S.G. Completely monotonic functions. Integral Transforms Spec. Funct. 2001, 12, $389-402$. [CrossRef]

13. Izumino, S.; Nakamura, N. Elementary proofs of operator monotonicity of some functions. Sci. Math. Jpn. 2015, 77, 363-370.

14. Coddington, E.A.; Levinson, N. Theory of Ordinary Differential Equations; McGraw-Hill: New York, NY, USA, 1955.

(C) 2020 by the authors. Licensee MDPI, Basel, Switzerland. This article is an open access article distributed under the terms and conditions of the Creative Commons Attribution (CC BY) license (http:/ / creativecommons.org/licenses/by/4.0/). 